\title{
Towards a better way of computing: a return from bits to atoms via physics and biology
}

\author{
Foreword to the first special issue on bio-inspired computing
}

\author{
Mihaela Ulieru
}

Published online: 10 November 2011

(C) Springer-Verlag 2011

Several emerging technologies related to the pervasiveness of computing, combined with advances in nano- and biotechnology, enable spontaneous linkages between people, systems and infrastructures to create "living" ecosystems that can configure and reconfigure themselves as needed. Such progress in computing has transformed our world to the extent that it can be argued that a very large number of entities now exist virtually in a universe of networked information, with distinct parallels to the universe we can normally apprehend through natural and extended human senses. These novel computing systems are bridging the physical and the virtual, merging us with our "things", transforming the way we live and work, and augmenting our abilities in unprecedented ways. Examples range from smart homes, grids and cities to self-reconfiguring manufacturing plants and self-deploying emergency task-forces, all relying on myriad sensors, mobile devices, software agents and human users that would build an ecosystem bringing the right skill, tool, or competence at the right time for the task at hand on the sole basis of local rules and peer-to-peer communication. Specifically, production processes are information-rich, and the dynamics of the information infrastructure is the tool for carrying it out both at individual locations and across the global environment. The electronic linking implies that work matter (or critical parts of it) is being transferred across virtual locations through a process which requires the intimacy between the physical and the virtual worlds to be restored.

"What if computation likewise respected physics so that it was possible to zoom from software to hardware without changing geometry and they scaled in exactly the same way?" In the first paper of our Special Issue Neil Gershenfeld addresses this question and its implications for the design of a novel computing that is aligned with the physical world, which promises to solve several problems, among which: scaling

M. Ulieru $(\varangle)$

IMPACT Institute for the Digital Economy, Ottawa, Canada

e-mail: ulieru@unb.ca 
information technologies, divergent chip design costs and challenges in multicore compilation.

In a similar attempt to bridge the real and the virtual, Giovanni Squillero considers artificial evolution in computer aided design to foster the intimacy between software and hardware when designing and testing microprocessors 'on silicon' by considering complex hardware characteristics, and shows how this can highlight very specific criticalities otherwise impossible to spot.

The demand from industry for novel and more effective ways of optimising design, and the response from academia which seeks to learn from biology about the mysteries of designing optimal systems with 'life-like' properties, is further exposed by James Whitacre in two articles. "Recent Trends Indicate Rapid Growth of Nature-Inspired Optimization in Academia and Industry" reveals the rapid growth in both demand and supply of novel bio-inspired approaches, emphasizing the need for the advanced research which will be hosted by our journal as a forum for exchange of latest original ideas and discoveries in this area. "Survival of the Flexible: Explaining the Recent Popularity of Nature-Inspired Optimization within a Rapidly Evolving World" deepens the investigation into the properties of life that we are seeking to the imitate in the quest to design systems and organizations with high agility, which can dynamically change, exhibit emergence, and self-organize to adapt, reconfigure and quickly respond to unexpected societal and environmental demands. Taking a look at today's digital ecosystems as an enabling technology for building the "backbone" and "central nervous system" of the future smart infrastructures that will enable the shift to a green and environmentally sustainable economy he further explains the recent increase in attempts to rephrase the classical concepts of engineering design and systems control respectively in terms of the evolvability and emergence found in natural systems, to develop technologies that can cope with the requirements of today's ever changing world.

The weaving of computing into every critical infrastructure at a global scale across regions, countries and continents tremendously exacerbates the risks threatening business continuity and public safety while at the same time becomes an opportunity to inject resilience and agility into critical infrastructures by designing the future networks with embedded safety and security mechanisms. Piet Van Mieghem takes inspiration from biological systems when investigating novel ways to contain the spread of malignancies in communication networks, while proposing an effective way to deal with the cascading effects of epidemics both in the virtual and physical realms.

This only opens the plethora of applications of bio-inspired computing spanning health, education and entertainment as well as energy, transportation, trade, commerce, and emergency response applications. Above all else, however, the enormous potential stems from the lessons that we can transfer from life's successful principles to revolutionize our governance structures to reset the current dynamics of our world from its perilous (market-and-conflict-oriented) trajectory, onto a prosperous one that is sustainable and focused on human needs. Please follow our Journal as we will continue to dedicate future Issues to each and every of these aspects of the exciting journey into the bio-inspired computing revolution. 